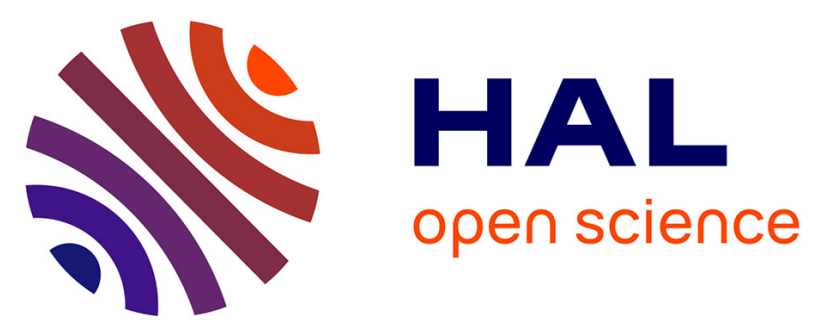

\title{
Performance of Context-Aware Publish/Subscribe systems for AANET
}

Mickaël Royer, Alain Pirovano, Fabien Garcia

\section{To cite this version:}

Mickaël Royer, Alain Pirovano, Fabien Garcia. Performance of Context-Aware Publish/Subscribe systems for AANET. Communication Technologies for Vehicles 8th International Workshop, Nets4Cars/Nets4Trains/Nets4Aircraft 2015, Sousse, Tunisia, May 6-8, 2015. Proceedings, 9066, Springer, pp 215-226, 2015, Lecture Notes in Computer Science, 978-3-319-17764-9 10.1007/9783-319-17765-6_19. hal-01337727

\section{HAL Id: hal-01337727 https://hal-enac.archives-ouvertes.fr/hal-01337727}

Submitted on 27 Jun 2016

HAL is a multi-disciplinary open access archive for the deposit and dissemination of scientific research documents, whether they are published or not. The documents may come from teaching and research institutions in France or abroad, or from public or private research centers.
L'archive ouverte pluridisciplinaire $\mathbf{H A L}$, est destinée au dépôt et à la diffusion de documents scientifiques de niveau recherche, publiés ou non, émanant des établissements d'enseignement et de recherche français ou étrangers, des laboratoires publics ou privés. 


\title{
Performance of Context-Aware Publish/Subscribe systems for AANET
}

\author{
Mickaël Royer, Alain Pirovano, and Fabien Garcia \\ ENAC Toulouse, France
}

\begin{abstract}
AANET (Aeronautical Ad hoc NETworks) are a subclass of Vehicular Ad-Hoc Networks (VANET). They are a very promising solution in order to answer to new air-ground communication needs. The Publish / Subscribe communication paradigm enjoys wide applicability in AANET where resources are limited. Many Publish / Subscribe systems exist for wired networks, however, these solutions are not fully adapted to mobile networks like AANET or VANET composed of cars. For the second category, the research community investigated and proposed some solutions. The aim of this article is to evaluate the performance of typical Publish / Subscribe systems suitable for VANET with cars on AANET with a realistic scenario based on commercial aircraft traffic. We show that some approaches suitable for VANET do not give good results in this case.
\end{abstract}

Keywords: Vehicular Ad Hoc Network (VANET), Aeronautical Ad Hoc Network (AANET), Publish / Subscribe communication systems.

\section{Introduction}

Mobile Ad-Hoc Networks (MANET) are self-configuring networks of mobile nodes connected by wireless links without a fixed infrastructure. Vehicular AdHoc Networks (VANET) are a subclass of MANET in which nodes are vehicle (cars, trains...). This sub-category shows a lot of specific characteristics, the most important being the high mobility of nodes and the predictability of movements. As detailed in [1, AANET (Aeronautical Ad hoc NETworks, which consist in aircraft acting as nodes of a MANET) can be seen as a subcategory of VANET. In a context of increasing air traffic and new needs in term of air ground communication systems, AANET represents a relevant solution as explained in [2]. Content-based information dissemination enjoys wide applicability in AANET where resources (like bandwidth) are limited. Notification of dangerous weather events to aircraft which will navigate close to the concerned geographical area or important messages from airline operational center to aircraft belonging to the company are good examples. Indeed, this approach allows a space decoupling between source and recipients since the sender of a message does not know which nodes will receive it. The aim of the network is then to find the recipients, based on the message's content. To accomplish content-based routing in a network, the Publish / Subscribe communication paradigm is the most widespread 
solution. In this model, event distribution from publisher (event producer) to subscriber (event consumer) is based on the subscriber interest and the event's content. Many Publish / Subscribe systems exist for wired network ([3, 4]) but are not suitable for MANET with low bandwidth and frequent network partition. Moreover, the applications described above need two extra features compared to classical Publish / Subscribe systems. The first one is the persistence of events in the network to reduce the problem of network partitions (similar principle as Delay Tolerant Networks, [5]). The second is the introduction of node context to reduce the scope of events/subscriptions and use more efficiently the network resources. As explained in [1, this context can be assimilated to a location context (an area or just a position). For example, with a classical Publish / Subscribe solution, aircraft can subscribe to "dangerous weather situation". However, this subscription will be too wide and will lead to the reception of useless events since aircraft are interested especially by "dangerous weather situation close to my position or route".

In the past years, some solutions have been proposed by the research community to provide Publish / Subscribe systems adapted to VANET (especially for cars), introducing the persistence of events and the use of node context in event delivery decision process. The aim of this article is to evaluate some solutions using different approaches against a typical scenario (notification of weather events) in a realistic AANET based on real traffic of commercial aircraft.

The next section presents a brief state of the art on Context-Aware Publish/Subscribe systems adapted for VANET. Section 3 compares some existing solutions based on a typical use case for AANET. The scenario and all implementation choices made to simulate aircraft mobility and Publish / Subscribe systems are explained in this section. Finally, section 4 concludes this article and introduces further work on this topic.

\section{Context-Aware Publish/Subscribe solutions in VANET}

As explained in [1, existing Context-Aware Publish/Subscribe systems that are adapted for VANET can be classified in three categories: geographic routing, proximity routing and overlay network based solutions.

Geographic routing based solutions like [6] or [7] are based on the assumption that each event / subscription is linked to a geographical area. The publish / subscribe system uses a geographic routing protocol to dispatch events from the publisher to the subscribers. Actually, an event is sent to the nodes located in the geographical area defined as the context of the event. In the same way, a subscription is sent to the geographical area defined in the context. Then, as described in figure 1a when a subscription matches with an event, nodes located at the intersection can act as middleman between publisher and subscribers.

Proximity routing based solutions like [8] or [9] provide many likeness with the previous solution. The main difference relates to the area where events and subscriptions are sent. This kind of solutions disseminate events/subscriptions only within a radius close to the sender (see figure $1 \mathrm{~b}$ ). 


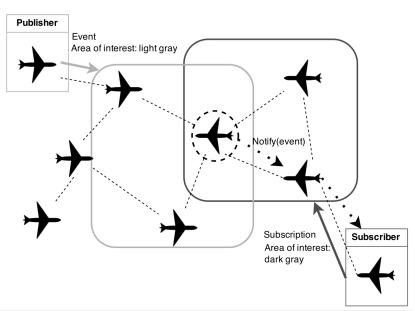

(a) Geographic

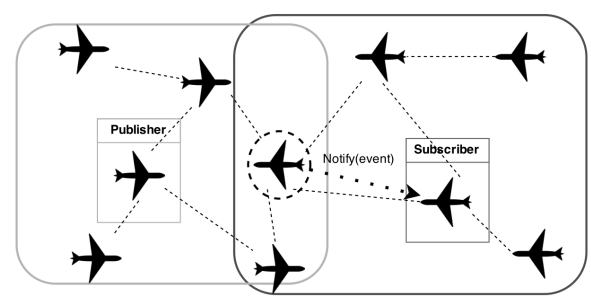

(b) Proximity

Fig. 1: Principle of geographic and proximity routing based $\mathrm{P} / \mathrm{S}$

Overlay network based solutions introduce a new component in $\mathrm{P} / \mathrm{S}$ architecture, the broker. Brokers are responsible for dispatching events from publishers to subscribers. They are interconnected and thus form an overlay network. In most of these solutions, like [10, brokers form an acyclic graph. Subscriptions are thus sent to all brokers of the graph (or just a part of it if context is used to filter recipient brokers). Based on these subscriptions, they can populate the table used to forward events to interested subscribers.

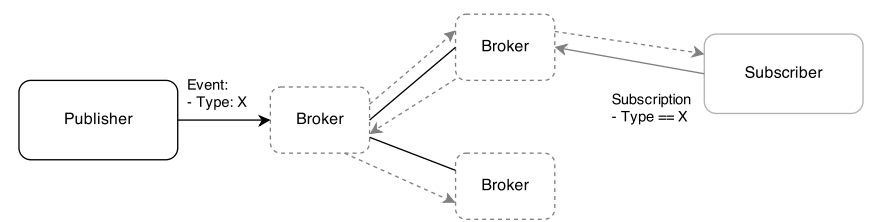

Fig. 2: principle of overlay network based $\mathrm{P} / \mathrm{S}$

\section{Solution comparison}

After a brief description of existing context-aware $\mathrm{P} / \mathrm{S}$ solutions, this section studies the performance of these solutions. Section A describes the scenario used to compare the solutions. This application relies on an AANET. Section B explains all the choices made during the implementation of the scenario and the $\mathrm{P} / \mathrm{S}$ solution in a simulation model. The last section explains the results obtained for each solutions.

\subsection{Scenario}

The scenario used to compare publish/subscribe solutions is a common problematic for civil aviation: how to inform aircraft of new weather situation ? Indeed, some phenomena like storm or wind shear are very dangerous and thus 
aircraft must be aware of these phenomena. Nowadays, these information are broadcasted by ground entities (like Air Traffic controller or airlines operational center) using radio, VDL (VHF Data Link) or SATCOM means.

A new approach to solve this problem can be found with the use of a context aware $\mathrm{P} / \mathrm{S}$ system over an AANET composed of commercial aircraft. In this scenario, aircraft aware of a dangerous phenomena, using its weather radar for example, sends an event over the network to announce the new weather situation. This event contains at least the type of event and the estimated duration of the event. The context associated to this event will be the geographical area cover by the weather phenomena. Meanwhile, aircraft know their short term trajectory and then can subscribe to weather events which happen near their route. The context associated to this subscription will be a geographical area that covers a part of the trajectory (see Figure 3).

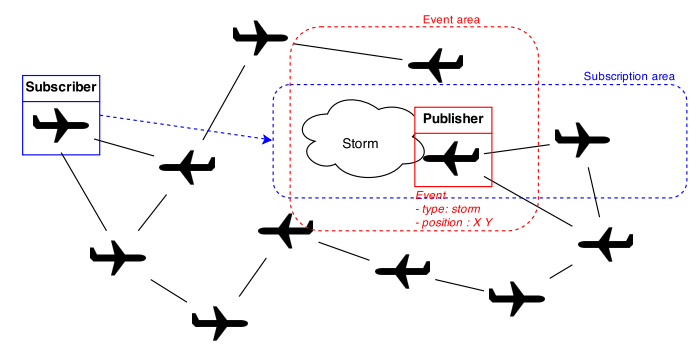

Fig. 3: principle of subscription to weather events

\subsection{Simulation / Implementation choices}

All the simulations have been performed with Omnet ++ ([11]). This paragraph deals with all the implementation choices that has been made to simulate the scenario and the Publish / Subscribe systems with Omnet ++ .

mobility The mobility model used for the simulations is based on real traffic traces from the French civil aviation authority and Eurocontrol based on either radar data or position report from the aircraft itself. Concretely, for a given day of the year, all position reports of commercial flights around the world (in spherical coordinate with an interval of $30 \mathrm{~min}$ ) can be obtained in a file following a specific format.

This file is then parsed by a tool developed at the ENAC, acparse. It offers many options to filter aircraft, interpolate trajectories and export the result to common format. In our case, acparse is used to get an XML file which contains two parts for each aircraft. The first one (surround by the tag mobility) contains position reports with an interval of 1 minute (thanks to the interpolation that 
acparse offers) and is used by a new homemade mobility module for Omnet ++ . The second one (surrounded by the tag plan) can be assimilated to a flight plan. In the simulation model, this flight plan is used by nodes in order to subscribe to weather event close to their short term trajectory.

Due to logistical limitations, it was difficult to simulate all the world traffic in reasonable time. As AANET is especially interesting for area where no ground infrastructures are available, as over oceans, we choose to reduce the scope to the transatlantic traffic. That is why our simulations are taking into account aircraft whose latitude belongs to the interval $\left[23,5^{\circ}-70^{\circ}\right]$ and the longitude belongs to the interval $\left[-90^{\circ}-10^{\circ}\right]$.

Physical/Mac layer Studies presented in this article deal with Publish / Subscribe service and not with the AANET feasibility which has been demonstrated in other studies like [2]. That's why it is not mandatory to have realistic physical and MAC layer. We choose to use IdealWireless module provided by the inet library [12], which simulates a simple physical and MAC layer. When a node sends a message, all nodes whose distance from the sender is below the parameter transmissionRange receive the message. In the same way, bit rate is a configurable constant. All messages are sent at this bit rate. However the values used for the simulations are deduced from a previous studies evaluating the feasibility of AANET built with commercial aircraft. In particular, [13] evaluates the use of CDMA as the layer 2 to interconnect aircraft and obtains an available bit rate of several Mbits/s for a transmission range of several hundred of kilometers.

Events / Subscriptions The generation of events meets the following rules. We fix the number of simultaneous events at the beginning of the simulation. We choose to keep this number low due to the size of the simulated AANET (limited to the transatlantic traffic). Each event occurs close to a randomly chosen aircraft. These events are then published by the aircraft close to it through the Publish / Subscribe system. When an event ends, a new event is created close to another aircraft and so on until the end of the simulation. The geographical area linked to an event is a cylinder which starts at the ground and finishes at an altitude of $20 \mathrm{~km}$ (the cruising altitude for a commercial aircraft is around $10 \mathrm{~km})$. The cylinder radius is a parameter of the simulation. The generation of subscriptions by aircraft is based on flight plan described in the section 3.2. This flight plan can be broken up to segments. Each segment fit 10 minutes of a flight and a subscription is generated for each segment. The time period associated to this subscription is the time interval between two position reports that form the segment. The geographical area linked to the subscription is the segment itself. Consequently, a subscription matches with an event if two conditions are verified. Firstly, the time period of the event fits the subscription's one. Secondly, the segment associated to the subscription intersects or is inside the cylinder which represents the geographical area of the event. 
$\mathbf{P} / \mathbf{S}$ Systems Three Publish / Subscribe systems have been simulated. The first one is the most simple. Events are disseminated to all nodes of the network. Subscriptions never leave the source node and are recorded in a table. When an event is received by a node, the event is checked against recorded subscriptions. If one matches, the event is forwarded to the application. This solution serves as a reference to compare performance of $\mathrm{P} / \mathrm{S}$ solutions and will be called "broadcast event solution" in the rest of this article. Intuitively, this solution gives the best result in term of delivery since all events are received by every node but is not resource efficient since even nodes that are not interested by an event receive it anyway.

The second one is a geographic routing based solution. LBM (Location Based Multicast) [14] is the geographical routing protocol used to dispatch events and subscriptions. LBM allows to send a packet to all nodes within a target geographic area (called a multicast region). In this way, the sender of the message defines a forwarding area according to his position and the multicast region. Only nodes within the forwarding area retransmit the message. Thus, the size of the forwarding area has a direct effect on the delivery ratio of the protocol (better with a large region) and the resulting load on the network (lower with a small region). The home-made implementation of LBM offers two options to define this forwarding region. The first one is explained in [14] and is based on the distance. A node $A$ forwards the message received from node $B$ for the region $R$ if $\operatorname{dist}(A, R)<\operatorname{dist}(B, R)+\delta$ where $\operatorname{dist}(A, R)$ is the distance from $A$ to $R$ and $\delta$ is a parameter of the protocol. The second option is a conic approach. In this case, $A$ forwards the message if $A \in \operatorname{cone}(B, R, \alpha))$ where $\operatorname{cone}(B, R, \alpha)$ is a cone whose the vertex is $B$, the axis is defined by the points $B$ and the center of the region $R, \alpha$ is the opening angle. This family of Publish / Subscribe solutions also requires a unicast protocol in order to send an event from the node used as an intermediary to subscribers interested by the event. In our case, we choose AODV 15 (Ad hoc On-Demand Distance Vector). This choice was motivated by the nature of the network (hundreds of highly mobile nodes) and the quality of service expected by the application. Our main objective is to be as efficient as possible in order to save bandwidth, furthermore, in this scenario, the delay to disseminate event to all subscribers is not a performance criteria. Thus a reactive routing protocol seems adequate and AODV is the reference in this category.

The third one is a proximity routing based solution. For each event/subscription, a proximity perimeter is defined (parameter of the simulation). The message is sent to all nodes inside this area. As with previous solution, when a node receives an event from which it knows subscribers, AODV is used to send this event to recipients.

\subsection{Results}

For all the results introduced in this section, the table 1 gathers the value of main parameters used for the simulations. The transmission range and bit rate chosen for the simulations are coherent with previous studies on AANET like [16. 
For each simulation, two results will be observed:

- Delivery ratio, for each event

$$
\text { Ratio }=\frac{\text { Number of subscribers that received the event }}{\text { Total number of subscribers }}
$$

- Total cost of network, which is the total number of bytes sent in the network (including control message) to dispatch events to subscribers. It is calculated as the sum of sent bytes (at the level of MAC layer) for all nodes of the network.

\begin{tabular}{|l|c|}
\hline Duration of the simulation & $24 \mathrm{~h}$ \\
\hline Day & $2012 / 12 / 25$ \\
\hline Transmission range & $300 \mathrm{~km}$ \\
\hline Physical layer bit rate & $1 \mathrm{Mb} / \mathrm{s}$ \\
\hline Duration of an event & uniform $(1800 \mathrm{~s}, 3600 \mathrm{~s})$ \\
\hline Duration of subscriptions & $30 \mathrm{~min}$ \\
\hline Size of zone covered by an event & $250 \mathrm{~km}$ \\
\hline Size of a subscription message & 32 Bytes \\
\hline Size of a event message & 4096 Bytes \\
\hline Number of simultaneous events & 5 \\
\hline
\end{tabular}

Table 1: Common parameter values for all simulations

The first paragraph details some general results, valid for all the simulations and useful to understand other results. The three following paragraphs show and explain results for each simulated pub/sub solution.

General results Table 2 lists the number of events and subscriptions generated during the simulation. We observe there is far more subscriptions message than event. This is the consequence of the chosen scenario. Indeed, only one event is active at a given time, then few events have been generated during the simulation. On the other hand, aircraft generate subscription every 10 minutes. As there are hundreds of aircraft flying for several hours, the result is more than 20000 generated subscriptions.

\begin{tabular}{|l|c|}
\hline Number of events & 150 \\
\hline Number of subscriptions & 23978 \\
\hline
\end{tabular}

Table 2: Number of events and subscriptions

Figure 4 shows the number of flying aircraft in the targeted area during the simulated day. This number has a direct impact on the network connectivity and 
thus on the delivery ratio possible by the pub/sub solution. We see number of aircraft during a part of the night is low compared to rest of the day. Consequently, it is difficult to obtain a delivery ratio close to $100 \%$ since it seems obvious that during this period, network partition exists. Previous studies on AANET like 2 found a reachable packet delivery ratio around $90 \%$. It appears to be the maximum possible value of delivery ratio reachable with a publish / subscribe solution.

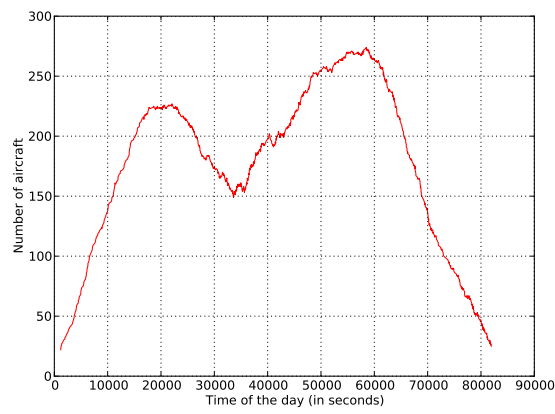

Fig. 4: Number of simultaneous aircraft during the day

Broadcast event solution This paragraph deals with results obtained with the event based dissemination solution. The table 3 shows the delivery ratio and the network load obtained with this solution.

\begin{tabular}{|c|c|}
\hline Delivery ratio & $64,02 \%$ \\
\hline Network & \\
\hline
\end{tabular}

\begin{tabular}{|l|l}
\hline Network load $3,70 \cdot 10^{7}$ \\
\hline
\end{tabular}

Table 3: Standard broadcast event solution

The observed result is not as good as estimated. However, it can be easily explained. The event has been disseminated in the network just after its creation. As the life of the event is between 30 and $60 \mathrm{~min}$, it is possible that an aircraft appears after the generation of the event but it is interested by it anyway. With the implemented solution, it can never received the event.

Figure 5 highlights this phenomena. We introduced a new parameter in the simulation: retransmiTime. If its value is strictly higher than 0 , it allows the retransmission of the event every retransmiTime seconds. The graphs show the obtained delivery ratio and network load depending on the retransmit time. We see delivery ratio can be increased up to 25 percent if the retransmit time is 
short (less than 600 seconds). The counter part is the exponential increase of the network load. As expected, this solution can give decent results at the cost of important network load which is not acceptable for AANET where bandwidth resources are very limited.

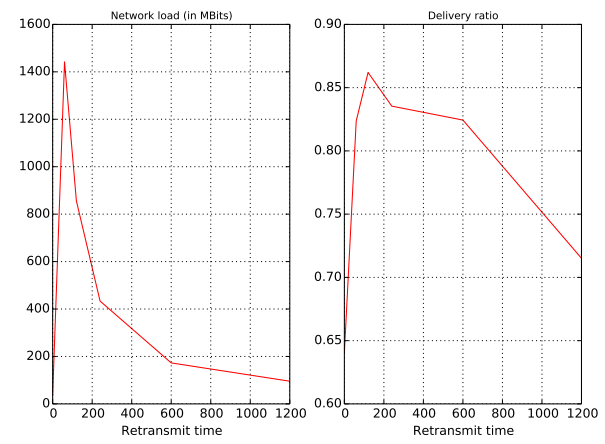

Fig. 5: Broadcast event solution depending on retransmit time

Geographic routing protocol based solution Results shown in this paragraph follow from simulations where LBM has been configured to use forwarding region based on the distance with $\delta=20 \mathrm{~km}$. Table 4 gives first results obtained with this solution. They are worse than expected. The delivery ratio appears lower than with the broadcast solution and most importantly the network load is higher. In conclusion, the broadcast event solution is more efficient than this one.

\begin{tabular}{|l|c|}
\hline Delivery ratio & $30,66 \%$ \\
\hline Network load & $7,16 \cdot 10^{8}$ \\
\hline
\end{tabular}

Table 4: Standard geographic routing based solution

Several reasons can explain this result. Firstly, events and subscriptions are only sent to nodes belonging to the area defined with the geographical context. In our case, it corresponds to the cylinder which represents the weather region for events and a cylinder that surrounds the aircraft trajectory for subscriptions. These regions are small thus few or no aircraft are present to act as middleman between publishers and subscribers. To highlight this problem, the model has been modified to extend geographic context area thanks to a new parameter, proximitySize which defines the radius of cylinder used as context region, the center being the center of the weather situation for events and the center of the 
segment for subscriptions. Figure 6 shows obtained results according to proximitySize's value. We see delivery ratio can be increased if the proximity size is extended but stays below $50 \%$ and thus this solution is far less efficient than the broadcast. The high network load can be explained by the characteristic of the application using the Publish / Subscribe service. As seen in section 3.3 aircraft send far more subscriptions than events and this solution forwards subscriptions compared to the previous one. Thus, even if subscriptions' size is lower than events' one, the important number of subscriptions increases substantially the load of the network.

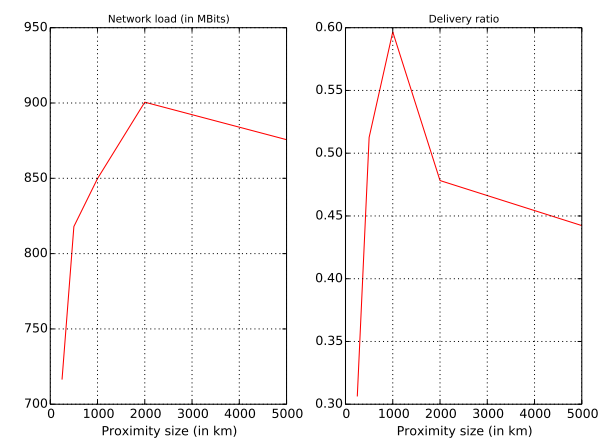

Fig. 6: Geographic routing based solution

proximity routing based solution Table 5 gives first results obtained with the proximity routing based solution (the perimeter has been set to $250 \mathrm{~km}$ ). These results are consistent with those obtained in the previous section. The delivery ratio is lower than with the geographic solution and the network load is roughly equivalent.

\begin{tabular}{|l|c|}
\hline Delivery ratio & $19,66 \%$ \\
\hline Network load & $4,54 \cdot 10^{8}$ \\
\hline
\end{tabular}

Table 5: Standard proximity routing based solution

In the same way than the geographic solution, we verify the influence of the delivery perimeter on the performance of the solution. Figure 7 shows results according to size of proximity area. As expected, the delivery ratio and the network load increase with the size of the proximity region. The maximum delivery ratio obtained is close to the one obtained with the broadcast event solution. However, the network load is higher. The reason is the same as earlier with the dissemination of subscriptions in the network. 

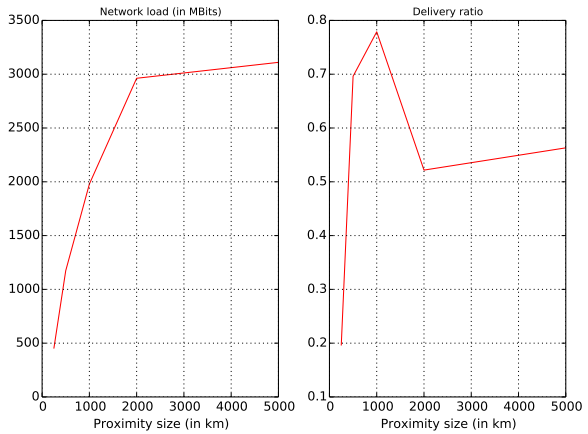

Fig. 7: Proximity routing based solution

\section{Conclusion}

In this article, after a brief survey about context aware Publish / Subscribe solutions adapted for VANET, we compare some solutions on AANET based on real commercial traffic, especially with a typical application for this type of network. We show that interesting approaches for VANET like geographical routing protocol are not suitable in this situation and lead to worse results than a basic approach based on the broadcast of the event in the network. Proximity based solution gives better results in terms of delivery ratio but with higher load of the network, which is not acceptable for AANET with low bandwidth resources.

Further work will concentrate on two axes. Firstly, this article shows that the development of a new publish / subscribe system is necessary to be suited to AANET and specific applications used on it. A promising approach is based on the definition of cluster in the network with different forwarding strategy inside and outside cluster groups. For example, we can imagine a solution where subscriptions are broadcasted inside a cluster group and events are sent to all cluster heads. It allows to take into account applications which generate far more subscriptions than events (as the one described in this article). The second axes is the research and the simulation of new application for which content based routing will be interesting. For example, in civil aviation domain, some research team studies ways to build real-time wind map and use of a Publish / Subscribe service over an AANET is a very promising solution to support this application.

\section{References}

1. Mickael Royer, Alain Pirovano, and Fabien Garcia. Survey on Context-Aware Publish/Subscribe Systems for VANET. In Communication Technologies for Vehicles, volume 7865 of Lecture Notes in Computer Science, pages 46-58. Springer Berlin Heidelberg, 2013. 
2. Frédéric Besse, Fabien Garcia, Alain Pirovano, and José Radzic. Wireless Ad Hoc Networks Access For Aeronautical Communications. In AIAA 28th International Communications Satellite Systems Conference, 2010.

3. Antony I. T. Rowstron, Anne-Marie Kermarrec, Miguel Castro, and Peter Druschel. SCRIBE: The Design of a Large-Scale Event Notification Infrastructure. In Proceedings of the Third International COST264 Workshop on Networked Group Communication, NGC '01, pages 30-43, London, UK, UK, 2001. Springer-Verlag.

4. P.R. Pietzuch and J.M. Bacon. Hermes: a distributed event-based middleware architecture. In Distributed Computing Systems Workshops, 2002. Proceedings. 22nd International Conference on, pages 611-618, 2002.

5. Zhensheng Zhang. Routing in intermittently connected mobile ad hoc networks and delay tolerant networks: overview and challenges. Communications Surveys Tutorials, IEEE, 8(1):24-37, quarter 2006.

6. I. Leontiadis, P. Costa, and C. Mascolo. Persistent content-based information dissemination in hybrid vehicular networks. In Pervasive Computing and Communications, 2009. PerCom 2009. IEEE International Conference on, pages 1-10, march 2009.

7. Davide Frey and Gruia-Catalin Roman. Context-aware publish subscribe in mobile ad hoc networks. In Proceedings of the 9th international conference on Coordination models and languages, COORDINATION'07, pages 37-55, Berlin, Heidelberg, 2007. Springer-Verlag.

8. R. Meier and V. Cahill. STEAM: event-based middleware for wireless ad hoc networks. In Distributed Computing Systems Workshops, 2002. Proceedings. 22nd International Conference on, pages 639-644, 2002.

9. P. Eugster, B. Garbinato, and A. Holzer. Design and Implementation of the Pervaho Middleware for Mobile Context-Aware Applications. In e-Technologies, 2008 International MCETECH Conference on, pages 125-135, jan. 2008.

10. Gianpaolo Cugola and Gian Pietro Picco. REDS: a reconfigurable dispatching system. In Proceedings of the 6th international workshop on Software engineering and middleware, SEM '06, pages 9-16, New York, NY, USA, 2006. ACM.

11. András Varga and Rudolf Hornig. An overview of the $\mathrm{OMNeT}++$ simulation environment. In Simutools '08: Proceedings of the 1st international conference on Simulation tools and techniques for communications, networks and systems 8 workshops, pages 1-10, ICST, Brussels, Belgium, Belgium, 2008. ICST (Institute for Computer Sciences, Social-Informatics and Telecommunications Engineering).

12. http://inet.omnetpp.org/.

13. F. Besse, A. Pirovano, F. Garcia, and J. Radzik. Interference estimation in an aeronautical ad hoc network. In Digital Avionics Systems Conference (DASC), 2011 IEEE/AIAA 30th, pages 4C6-1-4C6-11, Oct 2011.

14. Y.-B. Ko and N. H. Vaidya. Geocasting in mobile ad hoc networks: Locationbased multicast algotithms. In Proc. of the IEEE Workshop on Mobile Computing Systems and Applications (WM-CSA), 1999.

15. C. Perkins, E. Belding-Royer, and S. Das. Ad hoc On-Demand Distance Vector (AODV) Routing. RFC 3561 (Experimental), July 2003.

16. Quentin Vey, Alain Pirovano, Jose Radzik, and Fabien Garcia. Aeronautical Ad Hoc Network for Civil Aviation. In Axel Sikora, Marion Berbineau, Alexey Vinel, Magnus Jonsson, Alain Pirovano, and Marina Aguado, editors, Communication Technologies for Vehicles, volume 8435 of Lecture Notes in Computer Science, pages 81-93. Springer International Publishing, 2014. 